\title{
Ch. Bonnet, l'Abbé Clément et les Gordius*
}

\author{
Par R.M.Tecoz, Lausanne
}

Le Dictionnaire raisonné universel d'histoire naturelle de J.CH. VALmont DE Bomare est un ouvrage très souvent cité vers la fin du $18^{\mathrm{e}}$ siècle; in-8 ou in-4, il est peu encombrant avec ses six à douze volumes et il est économique d'autant plus qu'il n'a ni atlas de planches, ni figures. Son succès a été manifeste, ainsi que le montre cette remarque glanée dans une lettre inédite de Ch. Bonnet à Valmont de Bomare (du 24 juin 1775) : «... Non, je ne suis pas trop étonné de ces 30 mille exemplaires qu'on tire de vos ouvrages; ils sont du nombre de ceux qui doivent se trouver dans toutes les bibliothèques.»

La première édition de Paris eșt de 1765 (cinq volumes et un supplément); la deuxième a paru à Yverdon en 1768-1769 (douze volumes) et la troisième a été éditée à Lausanne par la Société typographique en 17761777 (neuf volumes) avec un supplément en 1778. Ce dernier a retenu spécialement mon attention car il a été rédigé par MrVICAT, D.M., le fidèle déchiffreur et traducteur de A. DE HALLER et médecin pensionné de la ville de Payerne.

Ce supplément comporte surtout une table «pour tous les objets d'Histoire naturelle, de physique et des Arts» et une autre «pour la Médecine, c'est-à-dire pour les vertus des médicaments, et pour les caractères des maladies...» avec un développement important des remèdes végétaux. Enfin, la dernière partie est faite: «Des articles retouchés ou nouveaux, tirés ou traduits des sources les meilleures et les plus modernes, et même des portefeuilles de plusieurs naturalistes des plus célèbres. »

Parmi ces articles, l'un d'eux m'a paru curieux; il se trouve sous la lettre G, aux pages 584-588, sous le titre «Gordius »; en voici la majeure partie, celle qui nous intéresse: «Un Ecclésiastique estimable, MrClement, administrateur de Champeri en Vallais, avoit cru découvrir que ce ver assez commun, nommé par les Naturalistes, Seta, Vitulus aquaticus \& par le peuple, fil de serpent, noissoit d'une Sauterelle.

„Ce ver, dit notre Prêtre, de différente longueur, mais d'une épaisseur toujours égale, quelquefois noir, quelquefois couleur-bai se trouve ordi-

\footnotetext{
* Je remercie vivement tous ceux qui ont facilité mes recherches: le Professeur R. MAtThey et ses collaborateurs à Lausanne; à Genève, Mademoiselle Emilie Trembley et le toujours si serviable archiviste Bernard GaGNebin.
} 
nairement dans les auges, les fontaines, les marais, souvent dans la bourbe des chemins; mais toujours dans l'eau douce et croupissante, n'est pas encore bien connu: son origine ne l'est pas. Linneus dit que sa morsure est dangereuse chez les Smolandois, et je l'ai toujours manié sans accident, peut-être parce qu'il était hors de l'eau sa tête est armée de deux pinces très visibles. Vu de loin, il ressemble à un gros crin de cheval. Souvent entortillé, c'est de-la que lui est venu le nom de poil ou soie aquatique. Des Paysans m'avoient dit l'avoir vu sortir du corps d'une sauterelle; je me défiai de leur observation; mais plus de soixante expériences faites avec soin me l'ont confirmée. La Sauterelle qui les produit, est la grande Sauterelle verte, décrite à la page 121 du huitième tome du Dictionnaire de Bomare, distinguée des autres par une épée ou une queue ensiforme. Le Gordius provient également du mâle et de la femelle de cette Sauterelle; j'en ai vu qui en étoient pleines; elles étoient encore farcies de leurs œufs ordinairement assez semblables à des grains de seigle. J'en ai vidé plusieurs de ces deux individus à la fois; c'est surtout dans le mois de Juillet qu'elles les déposent et toujours dans l'eau croupissante où quelquefois elles demeurent noyées; souvent on les voit pendre à l'anus d'un demi pied dans l'eau, ou dans un lieu humide où elles font lentement leur dépôt; ils y rentrent quelquefois assez vite quand on les éléve dans l'air. Il en est qui en recelent quatre ou cinq à la fois; leur couleur est différente comme leur maturité; les uns blancs, tendres et pelotonnés, n'ont que le tiers de leur épaisseur ordinaire, les autres sont plus ou moins noirs, et ceux-ci sortent les premiers. Cette maturité graduée prouve que la Sauterelle ne les a point avalés et ne les rend pas comme un aliment indigeste, qu'ils ne sont point le produit d'œufs étrangers éclos dans son sein; d'ailleurs j'en ai trouvé plusieurs à moitié murs encore attachés par l'une de leurs extrémités au placenta de la mère. Quoi de plus démonstratif et de plus propre à lever tous les doutes à cet égard?

»Lorsqu'ils ne sont pas mûrs encore, qu'ils sont inanimés et comme un peloton de fil blanc très délié, on les trouve toujours dans l'abdomen autour des intestins de la Sauterelle; mais devenus noirs et pleins de vie, ils se répandent jusque dans le thorax ou cavité de la poitrine. J'en ai tiré cinq d'une d'entre'elles que j'avois clouée dont j'avois ouvert le ventre, ils étoient de différentes grandeurs, plus de 30 heures après, pleins de vie et se débattoient ensemble comme des serpens sur une table ou dans l'eau. Le plus grand avait trois pieds et quelques pouces de long et je l'ai gardé deux mois dans une bouteille remplie d'eau. J'en ai gardé d'autres en diffé- 
rens tems, qui vivoient plus ou moins, quoique j'aie négligé de les changer l'eau ou d'y mêler du limon, ce qui peut-être eut prolongé leur vie.

"Sur la fin de l'automne, ils disparoissent; que deviennent-ils alors? J'ai conjecturé qu'il s'enfonçoit dans la terre ou la boue, où il se métamorphosse vraisemblablement en quelque autre insecte; ce qui le fait présumer, c'est qu'il n'en paroit point au printemps quelque beau qu'il soit, et qu'il ne paraît qu'après la ponte des Sauterelles...»

Cette découverte nous parut singulière [c'est VICAT qui parle] et nous crûmes devoir consulter un Naturaliste célèbre, Observateur exact et très ingénieux, et voici sa réponse.

"L'Auteur de la Contemplation de la Nature avoit beaucoup observé le Gordius en deux occasions différentes. Il avoit très-bien observé à la vue simple et à la loupe, les pinces ou crochets dont la tête est garnie et dont parle Mr. Clément. Cet Auteur avait surtout cherché à découvrir dans l'intérieur de ce ver singulier quelques vestiges de ces viscères qui sont si apparens chez la plupart des insectes, et quelque soin qu'il se fut donné, il n'avoit jamais pu parvenir à y rien démêler qui eut l'air vasculeux. Il avoit surtout été frappé des mouvements ondulatoires et continuels que son Gordius se donnait dans l'eau très-claire où il vivoit. Jamais l'Observateur n'avoit pu saisir un seul instant où ce ver ne fut pas en mouvement. Il avoit été pris dans l'eau d'une fontaine. Mr. DE SAussure l'observa plusieurs fois avec l'Auteur dont je parle, soit à la vue simple, soit à l'aide d'une très-bonne loupe, et il lui avoit paru comme à cet Auteur d'une substance partout uniforme. La tête, qui étoit très petite, étoit d'un brun noirâtre. Le corps tiroit sur le jaunâtre. L'intérieur étoit assés transparent.

"Qui l'auroit jamais cru que le Gordius fut le produit de la Sauterelle. Le Gordius n'est certainement pas le produit de la Sauterelle; mais il vit aux dépends de la Sauterelle dans laquelle il s'est introduit, comme d'autres vers mangeurs d'insectes vivans. La Sauterelle n'est donc point la mere du Gordius, comme le dit Mr.Clement; elle n'en est au plus que la merenourrice, et dans un sens qui n'est pas l'ordinaire.

»L'Observation des Gordius logés dans l'intérieur d'un insecte vivant, n'est pas nouvelle. Un grand Observateur, le célèbre DE GEER, avoit trouvé un de ces vers dans une teigne aquatique. Voyez ses Mémoires; Tom.II, Part.3, Planche 14, Fig.12, 13. Mais, Mr.Clément est, sans doute, le premier qui en ait trouvé dans les Sauterelles; et il est très-remarquable qu'il les y ait trouvé en aussi grand nombre. 
„D'ailleurs j'ai en ai trouve plusieurs à moitié murs, encore attachés par une de leurs extrémités au placenta de la mère; quoi de plus démonstratif et de plus propre à lever tous les doutes à cet égard? Cela seroit assurément très démonstratif, si le fait étoit bien constaté. La chose seroit encore des plus merveilleuses et d'un genre absolument nouveau. Mais Mr. Clément s'étoitil bien assuré, que ces jeunes Gordius étoient attachés par une de leurs extrémités au placenta de la mère? Un Insectologue demandera ici les preuves les plus rigoureuses; car il aura dans l'esprit cent et cent faits qui lui feront présumer des apparences trompeuses. On oseroit prédire à Mr. Clément, qu'un examen approfondi le détrompera. Il ne doit pas ignorer, que la génération des insectes est aussi régulière que celle des autres animaux.

"Tandis que ces vers sont encore inanimés; ils sont toujours animés, mais leurs mouvemens sont apparemment insensibles, ou presque insensibles dans leur enfance.

"J'ai gardé un Gordius plus de deux mois vivant dans une bouteille d'eau. L'Auteur qu'on a cité ci-dessus avoit fait la même expérience.

»Que deviennent-ils?... j'ai imaginé là-dessus une simple conjecture; c'est que le Gordius s'enfonce dans la terre, où il se métamorphose vraisemblablement dans quelque autre Insecte. On ne craint pas d'assurer à l'estimable Observateur, que le Gordius ne subit aucune métamorphose: au moins est-il bien sûr qu'on ne connoît aucun insecte de cette Classe, qui se métamorphose. On ne sauroit trop l'exhorter à pousser plus loin les curieuses recherches sur un ver si peu connu et si digne de l'être. Il faudroit surtout s'assurer s'il peut multiplier de bouture, comme ces vers longs d'eau douce, que le Contemplateur de la Nature à multipliés si prodigieusement en les coupant par morceaux.»

Parmi les questions que je me suis posées après la lecture de ces pages, la première que je veux examiner est celle de la priorité de ces observations; Mr.Clément, est-il comme l'écrit Сн.Bonnet en parlant des Gordius «sans doute, le premier qui en ait trouvé dans les Sauterelles»? Les nombreux traités généraux et modernes que j'ai consultés ne m'ont pas satisfaits; leurs remarques sont peu précises, fragmentaires aussi ai-je eu recours aux monographies; une des plus modernes et des plus importantes, celle de Dorier, ne traite pas l'historique du sujet. Parmi les ouvrages anciens, voyons le magnifique traité de DEGEer (cette orthographe est celle que l'auteur lui-même réclame!); on trouve, dans première édition, parue à Stockholm, au tome III (1773), p.432-433, le texte suivant: «Dans le premier volume de ces mémoires sur les Insectes (Mém.17, p.551, Pl.34, Fig.6 
et 7), j'ai parlé de certains vers blancs très déliés semblables à un crin de cheval et de la longueur de 3 pouces, qui vivent et se nourrissent dans le corps de certaines espèces de chenilles. Le 23 août, trois vers semblables sortirent devant moi du corps d'une grande Sauterelle de cette espèce (ce chapître est consacré à la grande Sauterelle verte); l'un de ces vers perça la peau près du derrière, les autres sortirent près du corselet. » La suite du texte précise qu'il s'agit bien là du «Ver-de-fil blanc des insectes (Gordius insectorum totus albus)...»; et plus loin: «M.Frisch parle aussi de semblables vers, qu'il a trouvés dans le corps des Sauterelles toutes vertes. J'ai fait voir ailleurs (...T.II, Mém.8, p.554) que le ver-de-fil ordinaire nommé Gordius... se trouve souvent dans le corps des larves de phryganes. » Dans le tome I du même ouvrage, Degeer écrit, à propos de la venue des Gordius dans la chenille de Zig-zac: «Ils n'ont pas été inconnus à Mr. Lyonnet, observateur célèbre, il en parle dans ses notes sur la Théologie des insectes.» (Il s'agit de la traduction en français de l'ouvrage de Mr Lesser, La Haye, 1742, 2 vol. in-8, tome I, note p.96). Dans l'édition en allemand des «Mémoires... » de K. Degeer (parue bien des années après l'édition en français), on trouve une note du traducteur, J.A.E.GöTzE, (tome III, p.280-281; Nürnberg 1780) qui se termine ainsi: «Vergleicht man die Würmer, die sich in den Insektenkörpern finden, mit den Gordius aquaticis; so wird man in Ansehung ihrer Bildung, Organen und Generation einen großen Unterschied finden und leicht überzeugt werden, $\mathrm{da} ß$ es eben sowohl, wie so viele andere Würmer thierischen Körper, davon man außer solchen Körpern keine examplare produciren kann, wahre Intestinal Würmer sind GöтzE». L'allusion de Degeer aux observations de J.L. Frisch, sans référence précise peut s'appliquer à deux travaux de ce savant; le premier en date est pour nous le plus intéressant; il est intitulé: «De lumbricis in locustis» et se trouve dans la publication: Miscellanea Berolinensia... continuatio III sive T.IV. Sumptibus Joh.Andr.Rüdigeri, 1734, p.393-396; en voici un extrait: "Anno 1727, cum aliquot Marchiae Brandenburgicae tractus locustis infestarentur... E plerisque... plures albos lumbricos, eos que sex, septem et plures pollices longos, et duas fere lineas lata... In media locustae alvo nidus horum vermium erat...» Je laisse de côté les considérations détaillées qui accompagnent ce texte. Le même observateur a fait une allusion à ses observations antérieures dans la XIIe Partie, p. 6 (1736) de sa Beschreibung von allerley Insecten... Le texte est bref; la seule désignation utilisée est «weiße Würmer» alors que dans le premier article, les parasites sont des «lumbrici...» 
Une recherche aussi complète que possible de la littérature spécialisée en diverses langues ne nous a pas montré des observations analogues antérieures à celles de J.L. Frisch qui paraît bien, sauf omission, être le premier à avoir publié une observation de ver du type Gordius* parasite des Sauterelles.

En ce qui concerne la Suisse, j'ai dépouillé bibliographies, livres, revues et compte-rendus des Sociétés savantes. Le premier texte que j'ai découvert se trouve dans la Bibliothèque universelle, tome $15,5^{\mathrm{e}}$ année, Décembre 1820; Sciences et Arts, Genève, p.288-294; c'est un article d'un médecin genevois, A.Matthey, établi à Paris, qui a relaté ses observations faites à Saint-Gervais: "On sait que le Dragonneau ou Gordius appartient à un genre - qui renferme plusieurs espèces - mais ce qu'on sait moins généralement peut-être, c'est que ce même ver - se trouve, dans nos climats, vivant dans le corps de la sauterelle verte, de la plus grande espèce (Locusta viridissima FABB.) » Les observations faites à Saint-Gervais ont montré, entre autres, la présence fréquente de ce ver qui peut sortir partiellement et rentrer par l'extrémité postérieure. Le reste de l'article traite du mode d'introduction des filaires dans les Sauterelles et surtout de la classification des diverses espèces. Notons, en passant, que c'est là l'unique article publié par ce médecin, insectologue d'occasion, sur une question d'histoire naturelle!

Seize ans après cette publication, dans les Acta S.S.S.N.1836, p.118, on note dans le compte-rendu des séances de la Société de physique de Genève (1 ${ }^{\text {er }}$ juillet $1834-30$ juin 1835): «Mr.F.J.PiCtet a montré un dessin représentant une filaire qui avait fait périr une Sauterelle en lui perforant son thorax de part en part.» Les Actes de la Société Helvétique des Sciences Naturelles de 1838 renferment un Rapport de la Société cantonale vaudoise (p.214): «Zoologie; Mr.BÉrANGer a présenté un ver intestinal vivant, de plusieurs pieds de longueur, trouvé dans la cavité abdominale d'une Sauterelle (Locusta viridissima); cet animal paraît appartenir au genre Filaire ou au genre Gordius.»

Il semble donc bien qu'on puisse considérer l'Abbé CLÉMent comme le premier observateur suisse ayant parlé des Gordius et des Sauterelles. Qui était donc cet Abbé Clèment ? L'Abbé J.M. Clément a vécu en Valais de 1736-1810; "vicaire d'Ardon, curé de Mage, recteur de Champéry puis vicaire d'Illiez. S'intéresse aux Sciences naturelles et à la Médecine; col-

\footnotetext{
* Le terme de Gordius s'applique aux Gordiacés en général; on sait que diverses variétés
} de ces vers ont été décrites depuis lors dans les Insectes, en particulier les Mermis. 
lections de botanique, d'insectologie, minéralogie» (notice biographique prise aux pages 324-326 de l'Essai d'Histoire de la vallée d'Illiez par les Abbés Damini et Deleze; un vol. in-8, Imprimerie Saint-Augustin à Saint-Maurice 1924); nous donnerons plus loin d'autres détails sur cet homme exceptionnel.

En lisant le texte du «Dictionnaire» de Bomare, la deuxième question que je me suis posée a été celle de l'identité du savant genevois auquel VICAT s'était adressé; la manière dont la réponse à la demande de VICAT est rédigée laisse perplexe; elle permet de supposer que ce n'est pas le savant auquel Vicat s'est adressé qui a écrit le texte cité. Quel était donc ce savant? Sans entrer dans le détail de nos recherches disons d'emblée que nous avons exclu Fr. Huber (1750-1831), Abraham Trembley (1710-1784) et que nous avons fini par penser qu'il s'agissait de Сн. Bonnet (1720-1793), bien que la manière dont il parle de lui-même nous ait paru curieuse.

Nous n'avions pas en mémoire le souvenir d'un passage des écrits de Ch. Bonnet faisant allusions aux Gordius et aux Sauterelles; à force de chercher nous avons fini par découvrir un texte révélateur dans le Tome IV, Partie II, des Euvres complètes (édition-4, Neuchâtel, 1781); dans la dixième partie de la Contemplation de la nature, au chapître XXXIII, à la page 168 débute une note 7, qui se rapporte au sujet traité: «celui des polypes et Animalcules desséchés qui reprennent vie... Le Gordius se trouve dans les fontaines et dans la terre, mais ce qu'on avait pas soupçonné, c'est qu'il se trouve aussi dans l'intérieur de quelques insectes vivants. L'illustre DE GEER l'avait trouvé dans une teigne aquatique; un Ecclésiastique estimable (en note: Mr.CLÉment, domicilié à Champéri dans le Valais) l'a rencontré fréquemment dans les Sauterelles. Il en a même trouvé plusieurs dans la même Sauterelle et qui n'avaient pris encore qu'une partie de leur accroissement...»

Ayant ainsi une quasi certitude, j'ai été à la recherche d'une éventuelle correspondance entre le Docteur Vicat, l'Abbé Clément et CH.Bonnet; j'ai trouvé seulement deux lettres de l'Abbé CLÉment et deux réponses de Ch. Bonnet dans les manuscrits et documents de ce savant à la Bibliothèque universitaire de Genève; il y a une allusion à ces lettres dans une note d'un article de C.. E. Engel (La Bibliothèque de l'Abbé Clément de Val d'Illiez, in La Suisse et ses amis, La Baconnière, u.d. (1943), un vol. in-8, p. 178-192).

La première lettre est datée du 2 Mai 1777; elle est un peu difficile à déchiffrer car les feuillets (2) ont été placés l'un sur l'autre avant d'être secs. 
Son contenu ne fait aucune allusion aux Sauterelles et aux Gordius; la lettre contient des remarques générales sur l'œuvre de Сн. Bonnet et sur d'autres sujets sans rapport avec le nôtre; j'en cite quelques extraits pour donner le ton général de ce début de correspondance. La réponse de Ch. Bonnet est datée: "A Genthod près de Genève le 31 e de Mai 1777 »; elle comporte environ trois-quart de page in-2 dont voici aussi les principaux passages.

\section{Lettre du 2 Mai 1777 de l'Abbé Clément à Ch. Bonnet.}

\section{Monsieur,}

Qu'un savant dont vous illustrez la classe, vous écrive, rien d'étonnant en cela; mais vous serez peut-être surpris qu'un étranger comme moi vous écrive: c'est votre célébrité et votre réputation universelle et si bien méritée qui en sont la cause. je ressens une estime et un respect tout particulier pour tous les vrais savants qui employent leurs talents d'une manière aussi utile que vous le faites, Monsieur. je ne vois rien, en fait de physique et d'histoire naturelle de plus exact, de plus clair, de plus méthodique et de mieux enchainé que vos excellentes productions dont j'ai vû une partie depuis quelque tems avec beaucoup de satisfaction.

Ne soyez point surpris, Monsieur, si je désire ardemment de voir la continuation de vos bons ouvrages, qui font les délices des savants; on y apprend à penser et à raisonner juste; on s'y instruit beaucoup. je n'écris point ceci pour vous éblouir par une poignée d'encens mais je parle comme je pense, en rendant justice à la vérité, d'ailleurs, je connais dans vos ouvrages mêmes votre grande humilité, qui n'en fait pas le moindre mérite: cependant je ne dis pas qu'il n'y ait rien à dire sur vos solides écrits... l'homme le plus habile ne sait rien faire d'absolumt parfait; mais étant plus fait pour faire goûter et admirer ce qu'écrivent les savant de bon et d'utile, que pour les critiquer, je me borne (en vous lisant toujours avec un nouveau plaisir) à vous demander si on aura bientôt une nouvelle édit: de votre considération sur les corps organisés, et précieux abrégé de physique, dont j'ai vû avec un plaisir extrême, une partie de l'édit: de 1768 (Amsterdam), mais sans avoir aucune réponse satisfaisante des Libraires, qui en annoncent une nouvelle, ce qui Monsieur, m'a fait prendre la liberté de m'adresser à l'auteur - vous-même. Seroit-il possible de vous procurer la moindre remarque utile, sur les matières que vous traitez si savamment?

Quoiqu'isolé dans une vallée de montagnes, privé de la société des savants, je me sens un goût et une inclination particulière, pour l'histoire naturelle. j'avais tenté, l'année dernière des expériences et observations relatives à celle de Mr. l'Abbé Spalanzani...» 
Je supprime la fin de cette lettre, qui ne concerne pas notre sujet.

Champery dans le Valais; Mr. Clément Prêtre.

$A^{\prime}$ Genthod près de Genève le $31^{\circ}$ de Mai 1777.

Je scavois, Monsieur, que les Alpes du Valais receloient un ami de la Nature. Je connoissois ses curieuses observations sur les Gordius qui se logent dans les Sauterelles, \& j'en avois dit ma pensée à un habile Naturaliste de notre Ville. Continuez, Monsieur, à étudier ces merveilles de la Création qui annoncent si hautement une INTELLIGENCE ORDONNATRICE. Vous ne pouvez trop feuileter ce Livre admirable exposé à tous les yeux, que trop peu de yeux savent lire, \& dont nous n'entendons encore que quelques paragraphes. Il est sur que les Salamandres reproduisent tous leurs Membres. Mon célèbre Ami Mr. SpaLLANZANi l'a démontré rigoureusement. Vous avez vu ce que j'en ai dit Part: IX, X de la Palingénésie philosophique. J'ai vu aussi un commencement de reproduction: mais un accident interrompit mon Observation. On ne se familiarise point avec de tels prodiges, \& l'homme pour qui ils seroient indifferens, ne seroit qu'un peu au-dessus du Singe. Le Libraire Ray d'Amsterdam, avoit déja publié deủx Editions des Cours organisés: il en publie à présent une troisième. Je n'y ai rien ajouté.

Chisol Libraire de notre Ville a la collection de tous mes Ecrits. J'ai fait dernièrement de nouvelles sur le Taenia. L'Abbé Rozıer les a publiées dans son Journal d'Avril. Elles contiennent des Faits intéressants, \& jusqu'ici inconnus aux Naturalistes. A'présent nous connoissons quelque petite chose sur cet étrange Animal. Je suis fort sensible au cas que vous voulez bien faire de mes petits Ecrits. J'en fais actuellement une Revision générale pour une grande Edition in $4^{\text {to }}-$ qu'un Libraire étranger veut en publier. Je tâche d'en diminuer le plus qu'il m'est possible les imperfections dont le nombre demeurera toujours trop grand. - Mais; ut potero. Cela ne paraitra guères que dans deux ou trois ans, \& peut-être plus tard.

Recevez les assurances de l'estime \& de la considération distinguée avec lesquelles j'ai l'honneur d'être, $\quad$ Mons $^{\mathbf{r}}$ Votre - - -

Ces deux lettres nous montrent que l'Abbé Chement ignorait que CH. Bonnet s'occupait de ses Gordius alors qu'avant cette date, Сн. Bonnet était déjà au courant des observations de notre insectologue valaisan! La deuxième lettre de l'Abbé CLÉment est datée du 25 septembre; j'en reproduit la plus grande partie (avec un fac-similé partiel ainsi que celui de l'adresse, intéressante, comme exemple des coutumes épistolières de ce temps-là); à la suite, la réponse de Bonnet à cette dernière missive. 


\section{Monsieur,}

Ayant appri que vous aviez fait la critique ou léxamen de mes foibles observations sur le Gordius, ce qui me fait autant d'honneur que de plaisir étant sur tout bien persuadé qu'elles n'auront d'autre mérite que celui de la nouveauté; à moin, que vous ne les approuviez: j'ai cru selon vos intentions, devoir multiplier les expériences, j'en ai fait encore un bon nombre, dans ce mois de septembre sur le Gordius qui m'étonne toujours, et me confirme cependant une bonne partie de ce que j'ai avancé, dans ma lère lettre, à Mrs. de la Société typographique; que l'on vous a communiqué à mon insu, et dont vous avez daigné faire plus de cas qu'elle n'en méritoit. je suis d'autant plus flaté que ma lettre vous soit tombée entre les mains, qu'il n'est certainement personne, sur tout en fait d'observat: plus capable que vous de discerner le vrai d'avec le faux.

Monsieur, la présente est pour vous offrir, si vous le souhaitez le petit Recueil de nouvelles remarques que je sors de faire sur le dit ver-Gord: je désire fort que celle-ci n'ait pas le sort d'une autre que jeus l'honneur de vous adresser, il y a près d'un mois et demi, sur certaines circonstances de la reproduction des pieds mutilés des Salamandr:aquat: que j'observe depuis quelque tems; je sens bien que vous ne l'aurez pas reçuë: car vous honnorâtes ma Ière $L$ : d'une réponse trop obligeante.

Outre les idées que je communique sur le papier, je crois qu'il est important de se communiquer encore le sujet même de ses éxpériences, (quand on peut), afin de se mieux entendre; C'est ce qui má déterminé, et fait prendre la liberté, de vous envoyer avec la présente, cinq sauterelles, de la véritable et seule espèce d'où je tire les Gord: il y a deux mâles et trois femelles, celles (comme vous savez qui ont la queue,(?) peut-être fais-je une folie d'envoyer des sauterelles à Genève, comme s'il n'y en avait pas; mais c'est à condition que vous ne soyez pas à portée d'en voir, et de les examiner.

Ces Sauterelles ne sont point toutes ni toujours pleines de Gordius, ce dont on ne peut s'assurer qu'après les avoir ouvert, il pourroit se faire que celles que je prends la liberté de vous envoyer, n'en auroient point; cependant je crois très fort qu'il s'y en trouvera, quoique peut être bien éloignés de leur maturité, car on les trouve souvent en pelotton aussi tendre et délié que celui des vers de la mouche à viande, quand on l'ouvre. etc.

Ces Sauterelles sont méchantes et vigoureuses, et quand elles peuvent se saisir, elles se déchirent impitoyablement, ce qui arriverait si elles forçoient les cloisons que j'y ai mis. je viens de les prendre toutes cinq aujourd'hui, en campagne, vers les 4 heures du soir. peut être que privées de nourriture elles pourroient mourir en chemin; cependant, quoiqu'elles mangent assez, j'en ai gardé, les 3 et même 4 jours, en vie, sans aucune nourriture. j'ai même tiré il y a quatre jours, un Gordius qui vit encore, d'une sauterelle qui avait été, au moins deux jours sans tête, sans être, entièrement morte. Si vous trouvez que les jambes sauteuses ou majuscules, manquent à quelques unes, cela nimporte, pour nôtre objet. 


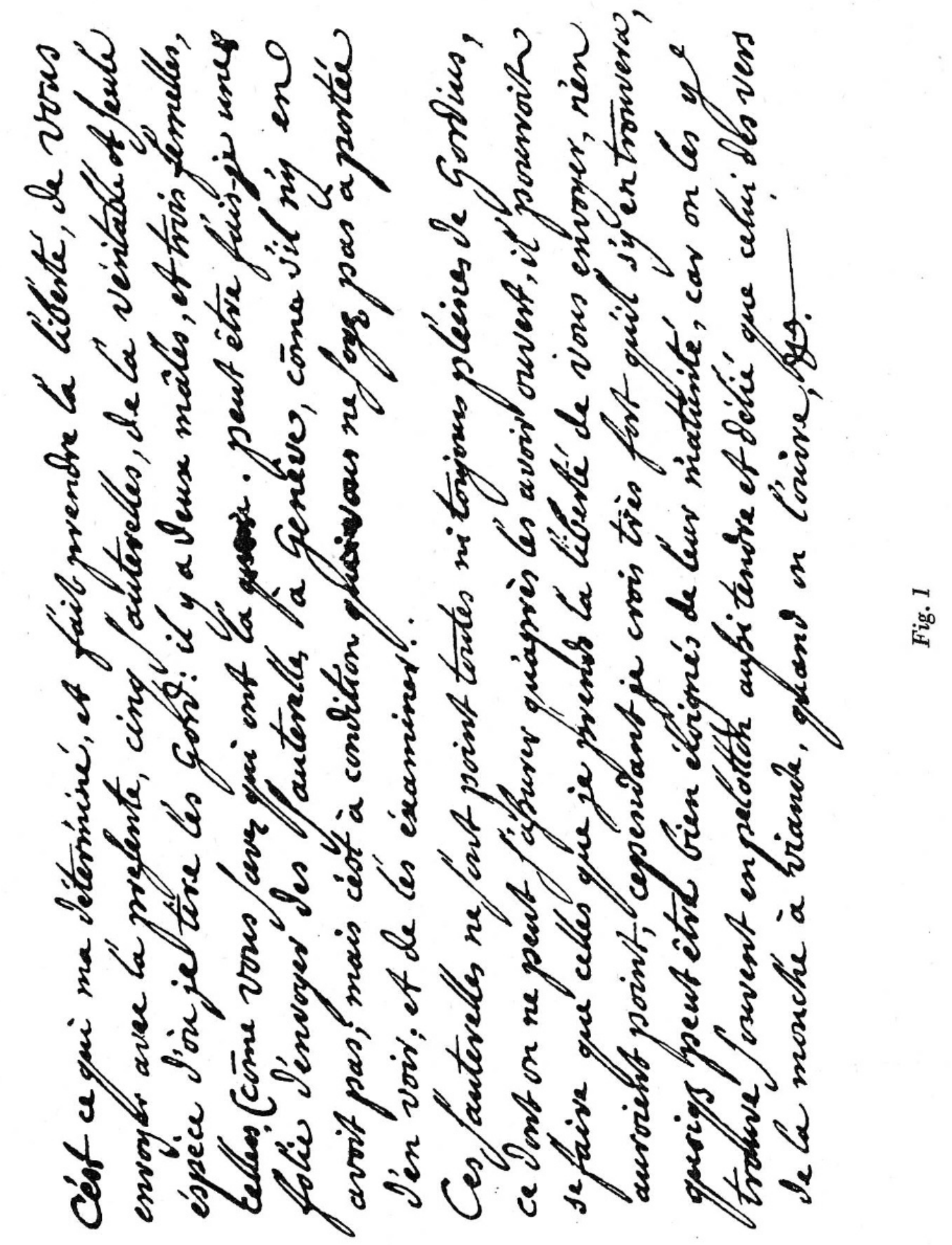


Si vous en voulez chercher ou faire chercher dans les prez, il faut toujours choisir les plus grosses et les plus pleines, alors elles manquent rarement de fournir des Gordius... Au reste, la femelle, (qua caudata est), parait souv: fort grosse, sans avoir autre chose, que les œufs de son espèce propre, qui sont en assez grand nombre.

j'aurais peut être pris la liberté, de joindre ici mes dernières observ: au risque de vous ennuier, mais je n'ai pu les transcrire; vous n'y perdrez guères : cependant, s'il en valloit la peine, je pourrais vous les faire passer par la voie de notre ami Mr. Vicat. en attendant, daignez pardonner mon importunité, et disposer de celui qui a l'honneur d'être avec beaucoup destime et la considération la plus distinguée

\section{Monsieur}

P.S. pourriez-vous

me procurer

quelques polypes d'eau

douce, dans une

piquette, etc. - - -

- - - - - -

de Champéry en vallais

25 septembre 1777.
Vôtre très humble et très obeis: serviteur j.M Clément prêtre administr.

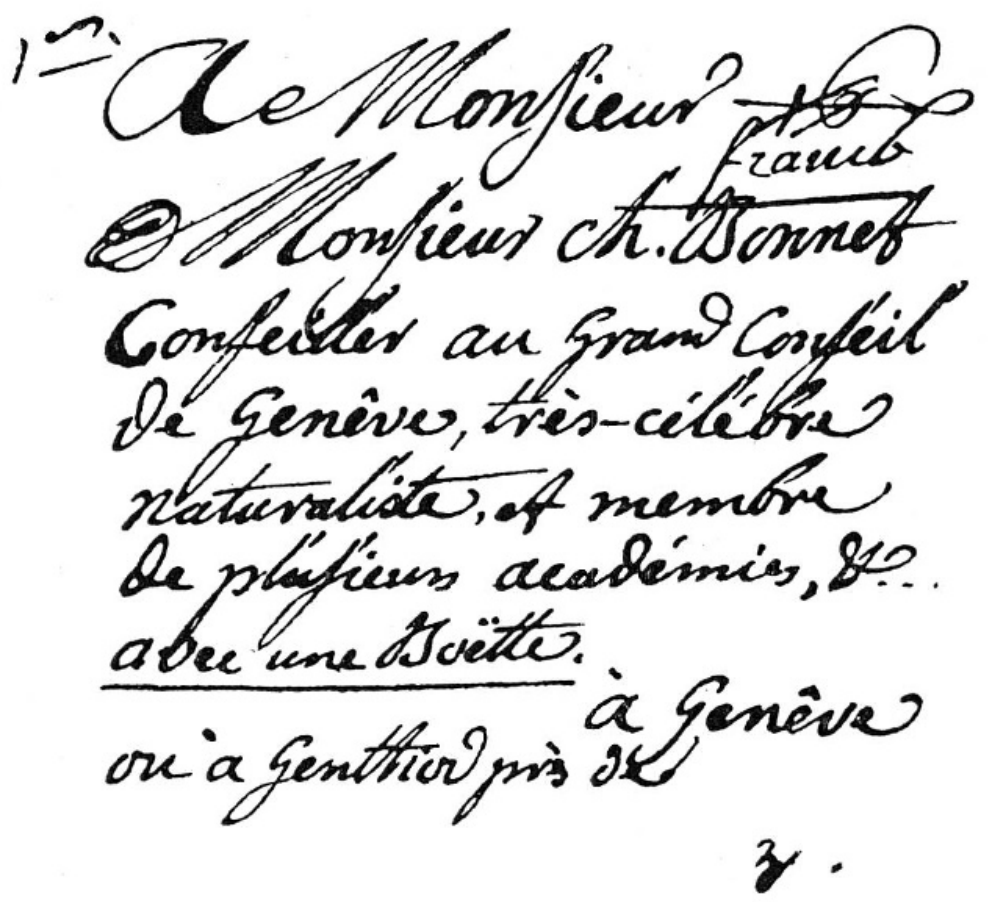

Fig. 2 
Champery dans le Valais, Mr Clément Prêtre

$\mathrm{A}^{\prime}$ Genthod près de Genève le $17^{\mathrm{e}}$ d'octobre 1777.

Je vous remercie, Monsieur, de l'envoi des Sauterelles. Les unes étaient mortes: les autres respiroient encore: mais, point de Gordius. Je persiste à vous le dire; je ne sçaurois croire que la Sauterelle soie dans aucun sens la Mère du Gordius. Il y a surement ici de l'équivoque ou des apparences déceptrices. Je me refère làdessus à ce que je vous ai fait parvenir.

Non, Monsieur; je n'ai point reçu cette Lettre où vous m'entreteniez des Salamandres. Je ne doute pas que vous n'ayaz vu la reproduction de leurs Membres. J'ai envoyé au Journal de l'Abbé Rozier un grand Mémoire sur ce beau sujet, ou je raconte la suite de mes Expériences. J'y ai joint des dessins, de la meilleure main, qui mettent sous les yeux du Lecteur les progrès de l'évolution. J'ai donc eu le plaisir de contempler de mes propres yeux cer merveilles du Monde organique, \& de confirmer ainsi l'intéressante Découverte de mon célèbre Ami Spallanzani. J'ai confirmé encore de la régénération de la tête du Limaçon, si contestée hors de l'Italie. Mon Mémoire sur cette admirable régénération a paru dans les Cahiers de Rozier de Septembre dernier.

Je suis fâché de n'avoir point sous la main quelques Polypes d'Eau douce. Ils ne sont pas rares dans les Fossés dont l'Eau se renouvelle. Ils ne vous échapperons pas, si vous prenez la peine de les chercher. Il suffiroit de prendre au hazard dans de tels Fossés quelques Plantes aquatiques, \& de les mettre dans un grand vase de verre plein d'eau claire. En regardant de temps en temps dans ce Vase, il pourra arriver facilement que vous y découvrirez des Polypes de plus d'un genre. Je suppose que leur description vous est connue.

Au reste, Monsieur; je vous conseillerais fort de publier dans le Journal de l'Abbé RozıeR* votre Découverte sur les Gordius. Vous exciteriez en leur faveur la curiosité des Naturalistes, et cela nous vaudra de nouvelles Vérités.

J'ai l'honneur d'être avec une parfaite considération, etc.

Maintenant que nous avons tous les documents disponibles sur le sujet qui nous intéresse, que pouvons-nous encore préciser en confrontant les textes imprimés et manuscrits.

Nous voyons que le DrVicat, au nom de la Société typographique, a soumis les observations de l'Abbé CLément à Ch. Bonnet mais il l'a fait par l'entremise d'un intermédiaire dont nous ignorons l'identité. C'est la raison pour laquelle nous ne trouvons aucune trace d'un échange direct de lettres entre Ch. Bonnet, le DrVicat ou la Société typographique; en

* Aucune trace d'un article de l'Abbé Clément dans ce journal. 
effet, les autres lettres de cette époque reçues par BonNEt ou les copies de celles qu'il a écrites ont été soigneusement conservées.

Quoiqu'il en soit, il semble que les relations de Сн.Bonnet et de l'Abbé Ciément en soient restées à ce bref échange de lettres et que l'offre de collaboration de l'Abbé Clément n'ait pas eu de suite. On ne peut s'empècher de penser combien ces rapports éphémères entre deux savants de valeur devaient avoir une importance bien inégale pour l'un et pour l'autre: l'Abbé CLÉment, autodidacte solitaire dans sa haute vallée et Сн. Bonnet, comblé par la renommée et la fortune mais néanmoins cruellement tourmenté par une perte progressive de la vision et un renoncement forcé aux joies de l'observation directe des insectes et des plantes.

Par cet exposé, j'espère avoir ajouté quelques données oubliées si ce n'est nouvelles à un des nombreux chapîtres incomplets de l'histoire des sciences naturelles dans notre pays. J'ai répété et confirmé que J.L. Frisch est le premier savant qui ait publié des observations de parasitisme des Sauterelles par les Gordius et ceci en 1734. En Suisse, c'est à l'Abbé Clément en 1777, que revient cet honneur avec une avance de quelque soixante ans sur les autres observateurs de notre pays. En faisant surgir du passé ces textes et ces observations oubliés et ignorés depuis presque deux siècles il m'a été agréable de rendre hommage à l'Abbé CLÉMEnT, «prêtre, administrateur de Champéry en Vallais», cet étonnant savant autodidacte, ami de la nature et des livres. 